\title{
Reply to Comment on "A dynamic rating curve approach to indirect discharge measurement by Dottori et al. (2009)" by Koussis (2009)
}

\author{
F. Dottori and E. Todini \\ Dipartimento di Scienze della Terra e Geologico-Ambientali, Università di Bologna, Bologna, Italy
}

\section{Introduction}

In a recent comment, Koussis (2010) moved a number of critiques on the paper by Dottori et al. (2009), which describes a methodology for indirect discharge measurement using simultaneous water stage measurements at two adjacent cross sections in a river reach. The procedure, which requires the geometrical description of the two cross sections, allows for direct computation of water surface slope, and improves accounting for unsteady flow effects through the use of the complete or simplified momentum equation (a reason for calling it DyRaC, the acronym of Dynamic Rating Curve).

Among others, Koussis argues that the procedure is not suited for practical use since it is too demanding in terms of data set and flow conditions, and the requirements for its application are generally not met in practice, particularly in rivers with a complex morphology. Then Koussis suggests using an alternative procedure for discharge measurement, based on a modification of the well known Jones Formula he developed several years ago (Koussis, 1975, 1976). Finally, he contends that the procedure proposed by Dottori et al. (2009) is based upon the well known "standard step method" for computing flood depth profiles.

The aim of this reply, is to show that the criticisms moved are not really motivated: DyRaC, the proposed approach, may be used for operational measurements in rivers with noticeable advantages with respect to alternative methodologies. As a matter of fact DyRaC has already been installed in Italy and thoroughly tested on-line for estimating unsteady discharges in real-time on the River Parma, as well as successfully applied for flow estimates on several gauging stations along the River Arno course.

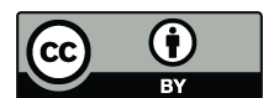

Correspondence to: E. Todini (ezio.todini@unibo.it)

\section{Koussis argumentation}

The criticisms and observations moved by Koussis to Dottori et al. (2009) paper, which will be referred to as DMT in the sequel, may be summarised into five points:

1. There seems to be an oversight in the sign of Eq. (4) of Dottori et al. (2009), for the celerity $c$ of the kinematic wave $(\mathrm{KW})$, which should be positive:

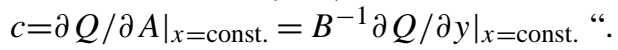

2. The DyRaC approach is costly and hardly applicable in practice.

3. DMT, in applying the Jones Formula, computed the celerity on the basis of the steady-state rating curve, while "when the flow departs markedly from the KW status, the Jones Formula should be evaluated with $c(Q)$ computed on the looped rating curve";

4. DMT's expectation of eliminating the extrapolation errors is overly optimistic;

5. DyRaC is nothing else that a reverse application of the standard-step method, an old procedure used by BGS (Darmstadt, Germany) to model flood flows in the River/Canal Kiphissos, in Athens, Attica Region, Greece (Koussis et al., 2003; Mazi and Koussis, 2006).

\section{Replies}

\subsection{On the error of sign in Eq. (4)}

The authors fully agree with Koussis that there is an oversight in the equation in DMT original paper. The authors are thankful to Koussis to give them the possibility of publishing a corrigenda of the mentioned error.

Therefore, Eq. (4) in page 849 in DMT paper must read $c=\frac{\partial Q}{\partial A} \cong \frac{1}{B} \frac{\partial Q_{0}}{\partial z}$.

Published by Copernicus Publications on behalf of the European Geosciences Union. 


\subsection{On the use of DyRaC methodology in practice}

According to Koussis opinion, the DyRaC methodology seems to miss some important and practical aspects of operational discharge measurement. First of all, he argues that the requirement of measuring water stage at two sections is not convenient from a practical point of view: (i) in order to have a good representation of water surface slope, which is necessary for the method to work properly, a careful placement of gauge stations is necessary, and "proper positioning of the two gauges is not a trivial requirement, because depth is controlled by the local stream geometry, in contrast to the flow rate that varies in space more gradually"; (ii) also, that "monitoring networks are shrinking worldwide and are increasingly difficult to maintain. Enhancing existing hydrometric networks with new stations is unlikely for reasons of cost".

Of course, the DyRaC methodology requires the geometrical description of two cross sections (one more than what is needed for a conventional rating curve), along with stagedischarge measurements for calibration, but the benefits in terms of accuracy are certainly rewarding.

As already stated in DMT, careful selection of the location where to install a flow measurement station is always needed; moreover, the river to be monitored must have good channel stability, without significant deposition and erosion processes, together with ease of access, in order to install and apply the necessary instrumentation. But these conditions are not only limiting the use of DyRaC, they also represent the essential requirements to the installation of a reliable conventional gauging station, where discharge is directly measured, or estimated by means of water stages and rating curves.

As opposed to what stated, but alas not really substantiated by Koussis, DyRaC can be successfully applied in practice because its requirements are much less stringent than what implied by Koussis in his comment. We can only agree when he states that an "engineering method is useful when it is theoretically sound and practically applicable. Practicability dictates that the essential prerequisites of a method should be readily secured." But we must disagree when he declares: "In the case of the DMT method, prerequisite is the existence of two appropriately positioned gauging stations, however, this may often not be the case." This could have been true in the past when water stage was essentially measured using floats, which implied the construction of complex measurement stations with the realization of "stilling wells" in which install the float. Today, ultrasonic and pressure type gauges can be easily installed. For instance, an ultrasonic water stage gauge can be installed on a bridge and a pressure sensor can be installed in the river bed $200 \mathrm{~m}$ upstream or downstream the first one.

As a matter of fact, the DyRaC methodology has already been applied with excellent results in real cases and under different conditions; an operational gauging station based on DyRaC is currently in use in the city of Parma (Italy), where two ultrasonic gauges were installed on two successive bridges, and double water stage sensors were also installed on several reaches of the river Arno in Tuscany (Italy) (Aricò et al., 2008; Dottori et al., 2008) in order to experiment the approach. For the Arno sections, more than 10 years of data are now available.

The authors are well aware that the DyRaC applications may be not possible in rivers located in impervious areas or characterized by strong sediment transport and/or braided channels. In such situations, a discharge estimation methodology needs to be based on simplifying assumptions and a limited amount of data to be collected. But this is not the field of application which the DyRaC methodology was designed for.

The main requirement for DyRaC to be successful is that under steady state conditions the water level difference results into the range of 2 to $5 \mathrm{~cm}$ : that is at least larger than the standard errors of water stage measurements (usually not larger than 1-2 cm). In contrast to what stated by Koussis, it is exactly because "depth is controlled by the local stream geometry in contrast to the flow rate that varies in space more gradually" that DyRaC will operate correctly. The flow between the two cross sections where the sensors are installed is in fact assumed to be constant when the reach is relatively short, but the variation of momentum is captured by the geometry of the cross sections and the measured water levels. This will also allow to capture backwater effects, similarly to stage-fall-discharge approach due to Herschy (1995), which can't obviously be captured by single water stage measurements.

A first order approximation of the length of reach needed can be roughly estimated as a function of the bed slope as in Table 1.

Although most rivers are characterized by bed slopes $>5 \times 10^{-5}$ (only very large rivers show milder slopes such as the Nile, which bed slope reaches $2.5 \times 10^{-5}$ ), from Table 1 one can see that problems to the practical application of DyRaC may arise when dealing with slopes smaller than $10^{-4}$. This is due to two reasons: the first problem is originated by the assumption of constant discharge in the reach, while the second one relates to the distance between the sensors, which does not allow the use of a unique data acquisition and transmission station.

As far as the first problem is concerned, this can be relaxed since a minor modification to the original DyRaC algorithm can easily take into account the change in storage in the reach due to the wave subsidence, thus allowing for a correct estimation of the discharge when the storage variation is not negligible. This modification and the relevant results will be presented in a forthcoming paper.

The second problem can certainly result into an economical problem: the new water stage sensors are relatively cheap, ranging from few hundreds to one thousand Euros, while data collecting and transmitting stations can be quite expensive. Their cost generally lies in the range of 
Table 1. Estimated reach length as a function of the river bed slope.

\begin{tabular}{ll}
\hline Bed slope $S_{0}$ & Distance $[\mathrm{m}]$ \\
\hline $1 \times 10^{-3}$ & $20-50$ \\
$5 \times 10^{-3}$ & $40-100$ \\
$2 \times 10^{-4}$ & $100-250$ \\
$1 \times 10^{-4}$ & $200-500$ \\
$5 \times 10^{-5}$ & $400-1000$ \\
$2 \times 10^{-5}$ & $1000-2500$ \\
$1 \times 10^{-5}$ & $2000-5000$ \\
\hline
\end{tabular}

10 000-15 000 Euros. Therefore, given that the distance for a reliable cable connection should not exceed 200-300 m, one can see that this is possible up to slopes of $10^{-4}$, where the data acquisition station can be placed between the two sensors, while larger distances inevitably imply a telecommunication link between one of the sensors and the data acquisition station. Therefore, on large and very mild rivers DyRaC may not be economically appealing as in steeper ones, but on such large rivers the cost of an additional station where the flow must be accurately determined (think of how important for Egypt is the correct estimation of inflows to lake Nasser) becomes irrelevant.

This allows us to discuss the second comment in which Koussis states that the networks around the world are shrinking and that it is unlikely that existing hydrometric networks will be enhanced for economical reasons. Although this is generally true in less developed countries, this is not the perception of these authors: high investments have been made in the last few years for the expansion and rehabilitation of existing networks in developed as well as in emerging countries facing flood problems. Just to give few examples in which one of the DMT author was involved: in Italy more than 1500 new multi-sensor telemetering hydro-meteorological stations were installed in the last decade; in Spain, several million Euros were spent on the rehabilitation of the SAIH, the Spanish hydrological information system; in China large investments were made in terms of equipment (telemetering gauges and meteorological radars) as well as flood forecasting modeling on the Yang Tse Jiang, the Yellow River and many other rivers; several rivers in Vietnam have been recently instrumented; the World Bank is funding a large telemetering network on the Mekong; The Sava River Authority is launching a major project for a new telemetering network on the Sava River; etc.

To conclude, we would also like to point out that, in order to implement DyRaC on existing stations, whenever this is possible from the hydro-morphological conditions as discussed above, it would be sufficient to install an additional water stage sensor and to connect it to the existing data collection station. This will generally result in a cost not exceeding 1000 Euros, which can be certainly afforded for the most important measurement sites.

\subsection{On the use of Jones Formula based on Koussis modification}

According to Koussis opinion, the original Jones Formula used in DMT is not the most appropriate formula to be compared to DyRaC suggesting that appropriately estimating the celerity on the loop rating curve, instead of estimating it on the normal or the steady state rating curve, can noticeably improve its performances and range of application.

First of all, the authors would like to clarify that the objective of DMT was to compare all the expressions for estimating unsteady flow rating curves available in the literature and, although not exhaustive, DMT incorporates a large number of them. Unfortunately, the Koussis proposed modification of the Jones Formula did not appear as an alternative to a rating curve estimation, but rather as a useful artefact to improve the performances of a kinematic wave (KW) model (Koussis, 1976).

Anyway, the authors think that the approach proposed by Koussis cannot be considered a practical and useful approach for estimating the unsteady flow discharges from water level measurements as will be demonstrated in the sequel.

It is well known that, in the absence of strong inertial effects and lateral inflow, a reasonably good approximation of the unsteady flow is provided by the convection-diffusion (CD) parabolic differential equation:

$$
\frac{\partial y}{\partial t}=D \frac{\partial^{2} y}{\partial x^{2}}-C \frac{\partial y}{\partial x}
$$

where $D$ is the diffusivity and $C$ the convectivity coefficient. Under the CD assumption (Weinmann and Laurenson, 1979) the loop rating curve can be approximated as:

$Q \cong Q_{s}(y) \sqrt{1-\frac{1}{S_{s}\left(Q_{s}, y\right)} \frac{\partial y}{\partial x}}=Q\left(y, \frac{\partial y}{\partial x}\right)$

with $Q_{s}(y)$ the discharge that can be derived from the steady state rating curve and $S_{s}\left(Q_{s}, y\right)$ the corresponding friction slope. Eq. (2) also shows that under the CD assumption, the unsteady flow can be reproduced as a function of the water level and of its spatial derivative. Jones Formula (1916) tries to approximate Eq. (2) as:

$Q \cong Q_{r}(y) \sqrt{1+\frac{1}{S_{r}\left(Q_{r}, y\right) U(y)} \frac{\partial y}{\partial t}}$

where $Q_{r}(y)$ is a "reference"discharge for the given stage $y, S_{r}\left(Q_{r}, y\right)$ is the corresponding "reference" water-surface slope and $U(y)$ is the surface velocity. For its practical utilization, Jones Formula requires the estimation not only of the reference rating curve $Q_{r}(y)$ (which for instance can be taken as the steady state discharge $Q_{s}(y)$ but also of the corresponding friction slope $S_{r}\left(Q_{r}, y\right)$ to be evaluated for a 
number of different flow conditions; this inevitably implies the use of more than one water stage measurement in space, at least in the calibration phase.

Henderson (1966) derived an alternative expression to the original Jones (1916) formula by replacing in Eq. (2) the space derivative as a function of the time derivative, namely:

$$
\frac{\partial y}{\partial x}=-\frac{1}{c_{K}} \frac{\partial y}{\partial t}
$$

where $c_{K}$ is the kinematic celerity. Note that Eq. (4) is only valid under the $\mathrm{KW}$ assumptions. Moreover, instead of the reference discharge $Q_{r}(y)$ used by Jones, following Chow's (1959) assumption of prismatic channel and uniformly progressive wave, Henderson used the normal flow $Q_{0}(y)$ in his new equation, which resulted in:

$$
Q \cong Q_{0}(y) \sqrt{1+\frac{1}{S_{0} c_{K}} \frac{\partial y}{\partial t}}
$$

Henderson (1966) correctly points out that these substitutions are possible "when subsidence can be neglected", namely when one makes the KW assumption and when the loop is not too wide. Under the same $\mathrm{KW}$ assumption by combining Eq. (4) with the continuity of mass equation, he also obtained an estimator for the KW celerity as:

$c_{K}=\frac{\partial Q_{0}}{\partial A}=\frac{1}{B} \frac{\partial Q_{0}}{\partial y}$

With reference to Eq. (5), there are three issues that it is worthwhile discussing. First of all Eq. (5) is generally misused in practice because while $S_{0}$ is assumed to be the bedslope (coinciding with the normal slope), the normal flow quantity $Q_{0}$ is usually evaluated using the steady state rating curve; but the $Q_{s}$ values along the steady state rating curve do not necessarily coincide with $Q_{0}$, because in natural rivers steady state and normal flow differ owing to the combination of the longitudinal variability of cross sections and possible backwater effects due to downstream conditions. The second and the third issues relate to the fact that although starting from the parabolic approximation, Eqs. (4), (5) and (6) are derived by imposing the KW hypothesis.

Consequently, when the spatial derivative of the water stage is substituted for in terms of the time derivative, the original CD approximation of the unsteady flow (capable of reproducing the wave subsidence as well as the loop in the rating curve), is forced back into a $\mathrm{KW}$ approximation. Moreover, the estimate of the kinematic celerity provided by Eq. (6) is additionally forcing the KW implication of no subsidence and no loop, given that its derivation implies again the same KW assumptions.

This was clearly understood by Perumal and Ranga Raju (1999) who pointed out that Eq. (5) can only be used to describe flood waves characterised by a "narrow loop", namely under conditions that do not depart much from the ones characterizing kinematic waves. And these are exactly the results that were found by DMT.

Koussis (1976) proposed modification aims at overcoming this KW forcing, by modifying the estimation of the kinematic celerity, which is now computed on the basis of the derivative of the actual discharge instead of the normal flow:

$c=\frac{\partial Q}{\partial A}=\frac{1}{B} \frac{\partial Q}{\partial y}$

and then substituted into Eq. (2) to give:

$$
Q \cong Q_{0}(y) \sqrt{1+\frac{B}{S_{0} \frac{\partial Q}{\partial y}} \frac{\partial y}{\partial t}}
$$

From a theoretical point of view, $c$ is no more the KW celerity $c_{K}$ (which must be estimated on the normal flow), nor the parabolic wave celerity (which is well known to equal infinity), nor the originally assumed CD equation (Eq. 1) convectivity coefficient $C$, which expression coincides with the kinematic celerity only when the wave subsidence effects are null. Koussis proposed modification is a practically oriented expedient to relax the KW assumption which can certainly be used to improve a simplified hydraulic model such as KCD. Unfortunately, as will be shown in the sequel, it also generates an instability problem that prevents its practical use as part of a discharge measurement system.

The loop rating curve, along which the celerity is now estimated, is no more a monotonically increasing function, such as the previously used normal flow curve or steady state rating curve. Therefore the celerity estimated using Eq. (7) presents two singular points, namely $c=0$ when $\partial Q=0$ and $c= \pm \infty$ when $\partial y=0$ as one can clearly see from the Fig. 2 of Koussis comment. Due to these singularities, the discharge estimates of Eq. (8) will also show singularities with $Q \cong+\infty$ when $c=0$ and $Q \cong Q_{0}$ when $c= \pm \infty$. This implies the need for introducing empirical subjective approximations in the range from $\partial Q=0$, corresponding to the maximum of discharge and $\partial y=0$ corresponding to the maximum of water stage. This fact is well known to Koussis who, in his original paper (1976) writes: "It must be noted here that in order to avoid numerical complications during the computation of the upper part of the rating curve (for example instabilities for $\Delta h / \Delta Q \rightarrow 0$ ), one should substitute there for $(\Delta h / \Delta Q)_{x}$ the gradient of the steady flow rating curve." Moreover, in his recent comment Koussis states "However, care must be exercised in the iterative calculation of $c$, to ensure convergence (Koussis, 1975; Weinmann, 1977; Weinmann and Laurenson, 1979; Ferrick et al., 1984; Perkins and Koussis, 1996)."

Unfortunately, the problem is far more serious than what seems to appear from Koussis statements. Weinmann, in his 1977 report quoted by Koussis, who reported some of its figures to support the quality of the Kinematic Model Corrected for Dynamic Effects (KCD) model, when commenting the $\mathrm{KCD}$ approach, specifically states, among others: 
"(iii) The iterative solution of the rating equation requires a number of restrictions on the value of the wave speed parameter, in order to prevent numerical instabilities near the tip of the loop. These restrictions have the secondary effect of reducing the accuracy of the solutions and it is difficult, if not impossible, to find a set of restrictions that provide high degree of both accuracy and numerical stability. The errors in the iterative computation of the rating curve produce a distortion of the outflow hydrograph. As a consequence the KCD model, unlike the other kinematic models, does not guarantee the conservation of mass."

Finally there is even a more interesting aspect that must be considered, which may explain why, within the context of a flood routing model, such as $\mathrm{KCD}$, the estimation of the rating curve may appear to be well performing.

Koussis (1976) in his KCD approach does not use $c$ or its inverse $1 / c$ but rather a spatial average $\langle 1 / c\rangle$ defined as:

“c) Instead of $(d A / d Q)_{x}=1 / c$ an averaged expression $(\langle\rangle)$ is introduced

$$
\left(\frac{d A}{d Q}\right)_{x}=\frac{1}{\Delta x} \int_{\Delta x}\left(\frac{d A}{d Q}\right)_{x} d x \equiv\left\langle\left(\frac{d A}{d Q}\right)_{x}\right\rangle \equiv\left\langle\frac{1}{c}\right\rangle "
$$

And further down in the same paper he explains:

"The stage hydrograph at cross-section $(j+1) \Delta x$ is obtained, also by an iterative computation of the pertinent unsteady-flow rating curve. Up to this point the computation can be considered as the first stage of an iterative routing procedure, which is to be continued. Now the gradient of the unsteady flow $A(Q)$ cures at the cross-sections $j \Delta x$ and $(j+1) \Delta x$ are available and hence an improved value for the parameter $\langle 1 / c\rangle$ can be determined. Herewith the flood is again routed. The computation yields an improved $Q_{(j+1, k+1)}$ value, which again leads to an improved stage $h_{(j+1, k+1)}$. Thus the gradient $(d A / d Q)_{x(j+1)}$ is again determined and evaluated for the computation of $\langle 1 / c\rangle$. This iteration can be carried through according to a preset limit of approximation. However, the improvement obtained finally will be generally of limited significance."

Therefore, in the KCD model, the modified Jones Formula is used as a point equation at one specific location (a cross section) as a "first stage of an iterative process", while the final result is based on spatial averages also including the successive cross section information. Which, at the end of the day, is what is suggested in DMT, namely to use the spatial information in order to capture the flood subsidence effects, which can hardly be discerned when uniquely using the time derivatives.

Despite all the above mentioned reservations, in order to test the validity of Koussis approach, and to evaluate its potentially practical utilization within the frame of an unsteady discharge measurement system, the authors have implemented Koussis suggested approach using the wave and channel characteristics indicated by Koussis in his comment on DMT (2010). This was made possible by Prof. Weine- mann, who in a personal communication provided the authors with the necessary information needed to set up the constrained solution of the resulting non-linear function, where the constraints aim at stabilizing the previously discussed instabilities. The results of the simulation, carried out at one single cross section, to maintain the spirit of Koussis comment, are not fully satisfactory as can be seen from Fig. 1 where the bed slopes of $2 \times 10^{-4}$ (left) and $1 \times 10^{-4}$ (right) where analysed. The estimation appears to be stable up to the proximity of the discharge maximum but, despite the use of constraints, substantial oscillations appear, which continue even after reaching the maximum stage. These oscillations are also influenced by the bed slope: one can notice that with a bed smaller slope the oscillations are larger.

\subsection{On the extrapolation of DyRaC beyond the range of measurements}

In his comment, Koussis argues that "no procedure based on the 1-D hydraulic equations, no matter how mathematically elaborate, can eliminate a judgment-based extrapolation of the rating curve beyond the range of actual measurements used for its derivation" and concludes that "DMT's expectation of eliminating the extrapolation errors overly optimistic."

In order to reply to this comment it is necessary to expand the statement contained in the conclusions of DMT, namely:

"Extrapolation beyond the range of measurements is essentially dominated by one parameter, an exponent, which controls the curvature of the rating curve; this produces a significant uncertainty in the extrapolation with large discharge estimation errors. On the other hand, in the DyRaC approach the curvature of the rating curve is correctly driven by the cross section geometry, which is known, while the evaluation of the roughness coefficient, which is the only required parameter, has a limited influence since it may be considered more or less constant at high flow regimes."

Traditionally, steady state rating curves are estimated by means of a monomial relationship, such as $Q=\alpha(y-\beta)^{\gamma}$, where $\alpha, \beta$ and $\gamma$ are the parameters to be estimated on the basis of couples of $y$ and $Q$, obtained via contemporaneous measurements of water depth and water velocity in the cross section of interest. Unfortunately, these values are seldom measured during high flows, which implies that the estimation of the parameters will only capture the low and medium flow values information, while the rating curve will be extended beyond the range of measurements. This "extrapolation" beyond the range of measurements causes one of the major discharge estimation errors (Di Baldassarre and Montanari, 2009), mainly depending on parameter $\gamma$, which controls the curvature of the rating curve. Another large error affecting discharge estimates is to neglect the unsteady flow effects, while smaller impacts on the flow estimation can be induced by other phenomena such as for instance the seasonality in the vegetation (Di Baldassarre and Montanari, 2009). 

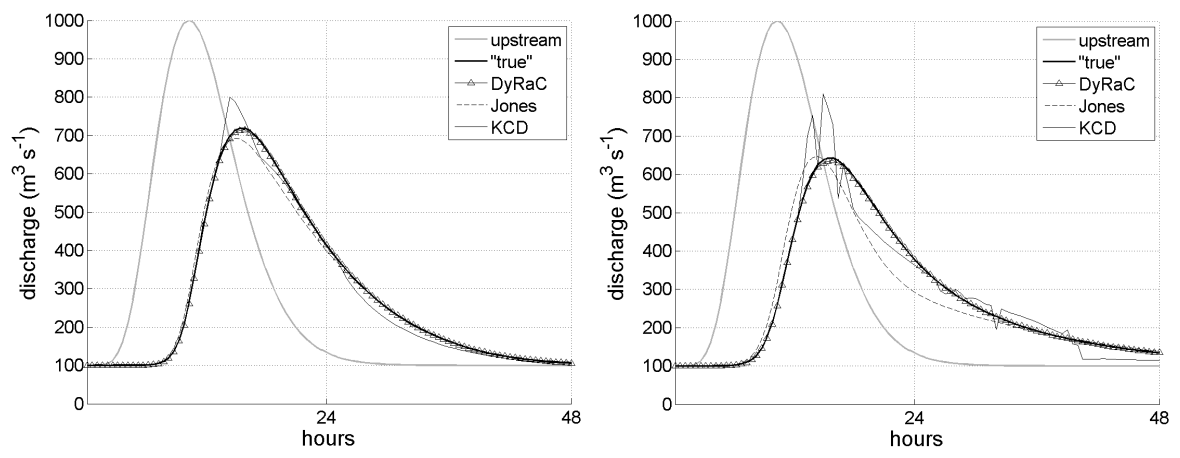

Fig. 1. Comparison of the discharge estimates based on DyRaC, Jones and KCD fromulas with the "true" values obtained using Hec-Ras (HEC, 2001). The left figure refers to the case of $2 \times 10^{-4}$ bed slope, while the right one to the case of $1 \times 10^{-4}$ bed slope. Note the instabilities present in the KCD approach.

The advantage of DyRaC over the conventional monomial equations representing the steady state rating curves is twofold. First of all it directly allows to estimate the unsteady flow discharge, thus eliminating one of the major sources of errors. In addition it offers another advantage in relation to the effect of extrapolation beyond the measurement range.

In order to explain this statement, let us take a simple trapezoidal cross section for the case of uniform flow. The relation between flow and discharge can be obtained as:

$Q=\frac{\sqrt{J}}{n} A R^{2 / 3}$

with $A$ the wetted area, $R$ the wetted perimeter, $n$ the Manning roughness coefficient, and $J=S_{0}$ the bed slope when dealing with uniform flow.

In the case of a trapezoidal cross section, this equation becomes:

$Q=\frac{\sqrt{S_{0}}}{n} \frac{B_{0}+B}{2} y\left(\frac{\frac{B_{0}+B}{2} y}{B_{0}+2 \frac{y}{\sin \theta}}\right)^{2 / 3}$

where $B$ is the surface width, $B_{0}$ is the bottom width while $\theta$ is the slope of the channel sides. When approximating Eq. (10) by the monomial expression, the curvature embedded in parameter $\gamma$, is not necessarily constant over the entire range. Parameter $\gamma$ essentially depends on the geometrical characteristics of the cross section and varies as a function of its shape.

This aspect can be clarified with an example, where the following values were used:

$S_{0}=2 \times 10^{-4} ; n=0.035 \mathrm{~m}^{-1 / 3} \mathrm{~s} ; B_{0}=20 \mathrm{~m} ; \sin \theta=0.196116$

where $\theta$ corresponds to a slope of the cross section sides of $1 / 5$.

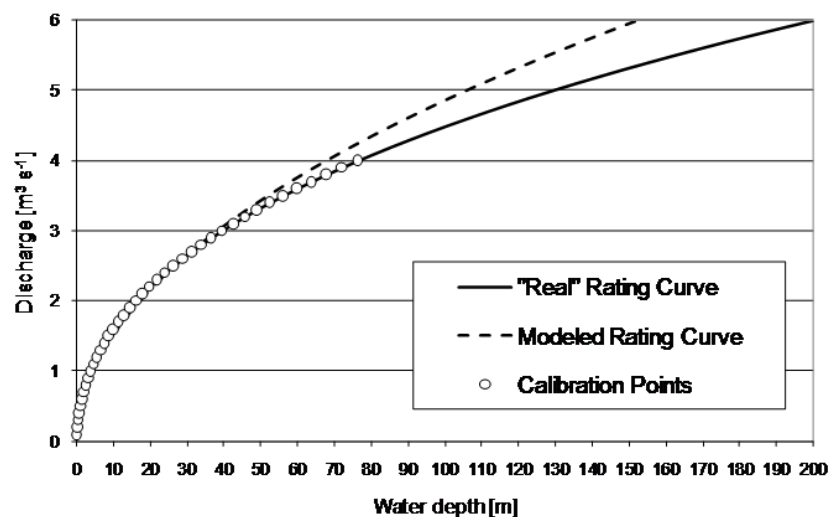

Fig. 2. An example of extrapolation using a monomial formula instead of the proper one of Eq. (10) in the case of a trapezoidal cross section. The dashed line is fitted to the "observations", the circles, supposed to be available for water depths smaller than $4 \mathrm{~m}$ and then extrapolate beyond.

The parameters of the monomial curve where estimated using water depths "observed" in the range $0-4 \mathrm{~m}$, by setting $\beta=0$ (not necessary in this case) and estimating the two remaining parameters via linear regression in the log space, to give $\alpha=4.211143 ; \gamma=2.0005$, with a regression coefficient $R^{2}=0.9974$, which indicates a reasonably good regression result. Nonetheless, as can be seen from Fig. 2, when extrapolating the curve beyond the range of observations, the difference between the values based on the monomial expression and the correct ones increases, with errors of the order of $24 \%$ when the water depth reaches $6 \mathrm{~m}$.

On the contrary in the DyRaC approach, which uses the actual cross section measurements, the geometrical characteristics are known. This allows to avoid the use of the traditional non-linear monomial expression approximation, a source of large extrapolation errors as shown above. 

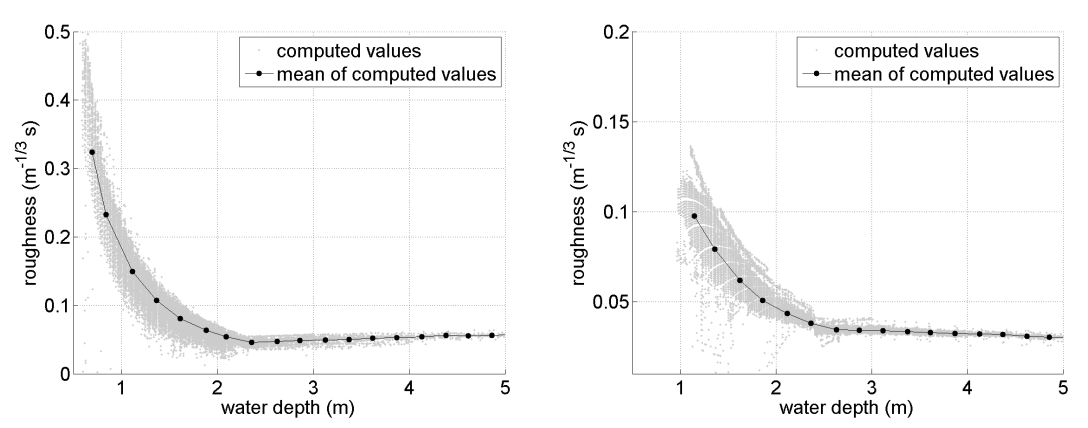

Fig. 3. Estimated roughness coefficients for the cross section of Incisa (left) and Nave di Rosano (right) in the river Arno. The variability of the roughness coefficient tends to vanish with increasing water depths.

On the contrary, DyRaC errors will mostly depend on the estimation of the head loss slope $J$ (which most of the times can be approximated by the water surface slope) as well as on parameter $n$, the roughness coefficient, and on its variability with the water depth. Fortunately, this dependence can be derived in calibration and only affects the computation at low values of $y$, because it tends to be asymptotically constant with increasing values of the water depth, as can be seen from the Fig. 3, where the estimated values of the roughness coefficient $n$ are plotted vs the water depth for the case of the river Arno in Italy. Figure 3 shows the shape of the estimated roughness coefficients in the cross section of Incisa (left) and Nave di Rosano (right) in the river Arno. As can be seen the variability of the roughness coefficient tends to disappear with depth.

It should be clear at this point that the meaning of the original statement, probably not sufficiently clear in DMT, was only meant to underline the above mentioned aspects of reduction of the large errors introduced by the monomial expression and the estimation of the curvature parameter instead of directly obtaining its shape from the geometrical description of the cross sections.

Having said that, we can fully concur with Koussis "that no procedure based on the 1-D hydraulic equations, no matter how mathematically elaborate, can eliminate a judgmentbased extrapolation of the rating curve beyond the range of actual measurements used for its derivation.” For example, in the case of sudden widening of a cross section where a berm is present, the dependence of the roughness coefficient on the water depth will increase again, which will therefore require an attentive analysis of the cross section as well as of its shape and vegetation in the calibration phase. The overspills, the complexity of river bed geometry, the presence of berms and vegetation will certainly affect the flow estimates. But this is invariably true whatever approach is taken and whatever steady state or looped rating curve approximation, is used. Therefore, although a careful analysis of the chosen site is necessary when establishing a water level and discharge measurement site, this does not prevent (as did not in the past) finding the appropriate and most suitable location which will try to minimise these errors. DyRaC has the merit of trying to eliminate the mathematical approximation, inevitably embedded into the used monomial expressions, which may result in large extrapolation errors.

\subsection{On the interpretation of $\mathrm{DyRaC}$ as a reverse standard step method}

Koussis contends "that the novel idea of DMT of using stage observations at two cross-sections to estimate flood flows and the BGS procedure of flood depth estimation from known flows are inversions of each other; indeed, both methods treat the flow as quasi-steady over the time interval for which the calculations are carried out. This standard procedure of BGS (Darmstadt, Germany) was adopted in the modelling of flood flows in the heavily modified Kiphissos (Kephisos) River, in Athens, Attica Region, Greece (Koussis et al., 2003; Mazi and Koussis, 2006)."

First of all Koussis seems to disregard the fact that two equations relevant to DyRaC were presented in DTM. The first one, Eq. (35), is derived from the full momentum Eq. (29), also including the local acceleration term, while Eq. (33), is derived from Eq. (28), where this term is neglected. It seems obvious that Eq. (35) cannot be classified as the "reverse" of the standard-step method.

Eq. (28), which can be used in the majority of cases, due to the fact that the local acceleration term generally plays a minor role, is the steady state momentum equation and its discretisation in space inevitably looks like the standard-step method equation (Eq. 30). The standard-step method was introduced by Chow (1959) as an alternative to the direct step method for drawing backwater profiles in a river, and successively also described by Henderson (1966), who termed it "step method." The standard step method was then widely used by one of the DMT authors to derive the PAB approach (Todini and Bossi, 1986) several years before the claimed standard BSG procedure applied to the Kiphissos (Kephisos) River, in Athens, Attica Region, Greece (Koussis et al., 2003; Mazi and Koussis, 2006). 
But the point is not this one. The actual point is that the DyRaC proposal may be defined as a "Columbus' Egg"1 (Benzoni, 1565). As a matter of fact, no one before DMT thought or proposed using the inverse of the steady (or unsteady) flow momentum equation to estimate the discharge given two water stage measurements. This is why DyRaC is "novel" and this is why it is also valuable because it is simple and can be practically implemented wherever one can use the standard step approach.

\section{Conclusions}

The authors would like to conclude by underlying that the alternative estimation of the unsteady flow in a cross section based on Koussis proposal does not meet the required stability standards and robustness needed to be used in practical applications, while, once again, would like to point out the advantages offered by the proposed DyRaC approach, which can provide a reliable solution in many practical applications.

\section{Appendix A}

\section{List of symbols used}

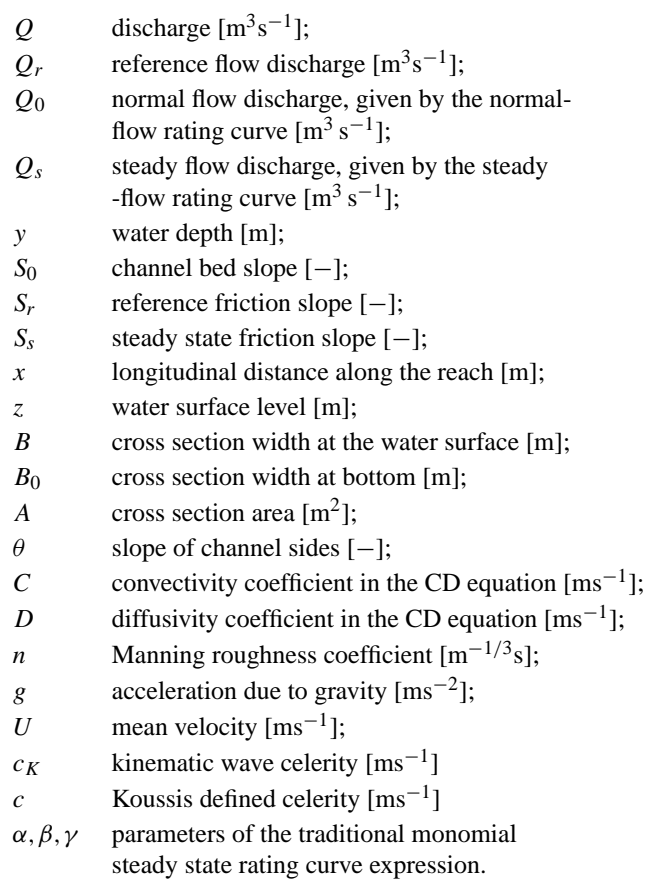

\footnotetext{
${ }^{1}$ An egg of Columbus or Columbus' egg refers to a brilliant idea or discovery that seems simple or easy after the fact. The expression refers to a popular story of how Christopher Columbus, having been told that discovering the Americas was no great accomplishment, challenged his critics to make an egg stand on its tip; and, after they gave up, he did it himself by tapping the egg on the table so as to flatten its tip (http://en.wikipedia.org/wiki/Egg_of_Columbus).
}

Acknowledgements. The authors would like to thank Muthiah Perumal and E. Weinmann for providing extremely useful information on the Koussis KCD (Kinematic wave Corrected for Dynamic effects) approach as well as on the constrained recursive estimation of the stage-discharge needed to implement it.

Edited by: F. Pappenberger

\section{References}

Aricò, C., Tucciarelli, T., Dottori, F., Martina., M. L. V., and Todini, E.: Peak flow measurement in the Arno River by means of unsteady-state water level data analysis, Proc. International Conference on Fluvial Hydraulics (RiverFlow), Cesme-Izmir, Turkey, 3-5 September, 2008.

Benzoni G.: Historia del Mondo Nuovo, Venice, 1565. English translation History of the New World by Girolamo Benzoni, Hakluyt Society, London, 1857.

Chow, V.-T.: Open-Channel Hydraulics - Mc Graw Hill - Tokio, 1959.

Di Baldassarre, G. and Montanari, A.: Uncertainty in river discharge observations: a quantitative analysis, Hydrol. Earth Syst. Sci., 13, 913-921, doi:10.5194/hess-13-913-2009, 2009.

Dottori, F., Martina., M.L.V., Todini, E.: Misure indirettedi portata in alvei naturali, Atti del XXXI Convegno Nazionale di Idraulica e Costruzioni Idrauliche, Perugia, Italy, 9-12 September 2008 (in Italian).

Dottori, F., Martina, M. L. V., and Todini, E.: A dynamic rating curve approach to indirect discharge measurement, Hydrol. Earth Syst. Sci., 13, 847-863, doi:10.5194/hess-13-847-2009, 2009.

Ferrick, M. G., Blimes, J., and Long, S. E.: Modeling rapidly varied flow in tailwaters, Water Resour. Res., 20(2), 271-289, 1984.

Henderson, F. M.: Open channel flow, Macmillan Series in Civil Engineering, The Macmillan Company, New York, USA, 1966.

Herschy, R. W.: Streamflow Measurement, 2nd edn., E\&FN Spon, London, UK, 1995.

Hydraulic Engineering Centre (HEC): Hydraulic Reference Manual, U.S. Army Corps of Engineers, Davis, California, USA, 377 pp., 2001.

Jones, B. E.: A method of correcting river discharge for a changing stage, U.S. Geological Survey Water Supply Paper 375-E, 117130, 1916.

Koussis, A.: Ein Verbessertes Näherungsverfahren zur Berechnung von Hochwasserabläufen (An Improved Approximate Flood Routing Method), Technical Report Nr. 15, Institut für Hydraulik und Hydrologie, Technische Hochschule Darmstadt, 1975 (in German).

Koussis, A.: An Approximative Dynamic Flood routing Method. Proc. Int. Symp. on Unsteady Flow in Open Channels, 1215 April 1976, Newcastle upon Tyne, England, Pages L1-L12, 1976.

Koussis, A. D., Lagouvardos, K., Mazi, K., Kotroni, V., Sitzmann, D., Lang, J., Zaiss, H., Buzzi, A., and Malguzzi, P.: Flood forecasts for an urban basin with integrated hydro-meteorological model, J. Hydrologic Engineering, 8(1),1-11, 2003.

Koussis, A. D.: Comment on "A dynamic rating curve approach to indirect discharge measurement"by Dottori et al. (2009), Hy- 
drol. Earth Syst. Sci., 14, 1093-1097, doi:10.5194/hess-14-10932010, 2010.

Mazi, K. and Koussis, A. D.: The 8 July 2002 storm over Athens: analysis of the Kifissos River/Canal overflows, Adv. Geosci., 7, 301-306, doi:10.5194/adgeo-7-301-2006, 2006.

Perkins, S. P. and Koussis, A. D.: A stream-aquifer interaction model with diffusive wave routing, J. Hydraulic Eng., 122(4), 210-219, 1996.

Perumal, M. and Ranga Raju, K. G.: Approximate convection- diffusion equations, J. Hydrol. Eng.-ASCE, 4(2), 160-164, 1999.
Todini, E. and Bossi, A.: PAB (Parabolic and Backwater), an unconditionally stable flood routing scheme particularly suited for real time forecasting and control, J. Hydraul. Res., 24(5), 405424, 1986.

Weinmann, P.E.: Comparison of Flood Routing Methods for Natural Rivers, Report No. 2/1977, Dept. of Civil Eng., Monash University, 182 pp., 1977.

Weinmann, P. E. and Laurenson, E. M.: Approximate flood routing methods: A review, J. Hydraul. Div. ASCE, 105(12), 1521-1536, 1979. 